\title{
Analysis of the genome content of Lactococcus garvieae by genomic interspecies microarray hybridization
}

\author{
Mónica Aguado-Urda', Guillermo H López-Campos², José F Fernández-Garayzábal ${ }^{1,3}$, Fernando Martín-Sánchez², \\ Alicia Gibello¹, Lucas Domínguez ${ }^{3}$, María M Blanco ${ }^{1 *}$
}

\begin{abstract}
Background: Lactococcus garvieae is a bacterial pathogen that affects different animal species in addition to humans. Despite the widespread distribution and emerging clinical significance of L. garvieae in both veterinary and human medicine, there is almost a complete lack of knowledge about the genetic content of this microorganism. In the present study, the genomic content of L. garvieae CECT 4531 was analysed using bioinformatics tools and microarray-based comparative genomic hybridization (CGH) experiments. Lactococcus lactis subsp. lactis IL1403 and Streptococcus pneumoniae TIGR4 were used as reference microorganisms.

Results: The combination and integration of in silico analyses and in vitro CGH experiments, performed in comparison with the reference microorganisms, allowed establishment of an inter-species hybridization framework with a detection threshold based on a sequence similarity of $\geq 70 \%$. With this threshold value, 267 genes were identified as having an analogue in L. garvieae, most of which $(n=258)$ have been documented for the first time in this pathogen. Most of the genes are related to ribosomal, sugar metabolism or energy conversion systems. Some of the identified genes, such as als and mycA, could be involved in the pathogenesis of L. garvieae infections.
\end{abstract}

Conclusions: In this study, we identified 267 genes that were potentially present in L. garvieae CECT 4531. Some of the identified genes could be involved in the pathogenesis of $L$. garvieae infections. These results provide the first insight into the genome content of L. garvieae.

\section{Background}

Lactococcus garvieae is one of the most important bacterial pathogens that affect different farmed fish species in many countries, although its major impact is on the trout farm industry [1,2]. In addition to farmed fish, this microorganism has also been isolated from a wide range of wild fish species, from both fresh and marine water, as well as from giant fresh water prawns [3] and from wild marine mammals [4]. The host range of L. garvieae is not limited to aquatic species. This agent has also been identified in cows and water buffalos with subclinical mastitis [5,6] and from cat and dog tonsils [7]. In humans it has been isolated from the urinary tract,

\footnotetext{
* Correspondence: mmblanco@vet.ucm.es

'Departamento de Sanidad Animal, Facultad de Veterinaria, Universidad
} Complutense, 28040 Madrid, Spain blood, and skin and from patients with pneumonia, endocarditis or septicaemia [8-11]. Recently, intestinal disorders in humans have been associated with the consumption of raw fish contaminated with this pathogen [12], which suggests that $L$. garvieae could be considered as a potentially zoonotic bacterium [3,12]. Despite the widespread distribution and emerging clinical significance of $L$. garvieae in both veterinary and human medicine, there is almost a complete lack of knowledge about the genetic content of this microorganism.

In the last few years, research in microbial genetics has changed fundamentally, from an approach involving the characterization of individual genes to a global analysis of microbial genomes. The availability of complete genome sequences has enabled the development of high-throughput nucleic acid hybridization technologies including macro- and microarrays. Microarrays have the

\section{() Biomed Central}


capacity to monitor the genome content of bacterial strains or species very rapidly. Although whole-genome sequencing is definitely a powerful method for genetics, it is still expensive and time consuming. As an alternative, comparative genomic hybridization (CGH) experiments based on microarrays have been used to facilitate comparisons of unsequenced bacterial genomes. Arraybased CGH using genome-wide DNA microarrays is used commonly to determine the genomic content of bacterial strains [13,14], but also for inter-species comparisons [14-16]. In this case, microarrays of closely related microorganisms that have been fully sequenced must be available. The primary advantage of this microarray approach is that it allows the identification of a large number of genes that are potentially present in an organism without the need for sequencing genomes. The disadvantage of this approach is that it indicates only the genes that are common between the fully sequenced relative and the strain of interest; genes unique to the strain of interest remain unknown $[15,17]$. In the present work the genetic content of $L$. garvieae CECT 4531 was studied by a combination of in silico analysis and in vitro microarray CGH experiments, using open reading frame (ORF) microarrays of two bacteria closely related to L. garvieae, namely Lactococcus lactis subsp. lactis IL1403 and Streptococcus pneumoniae TIGR4 $[18,19]$.

\section{Methods}

\section{Bacterial strains, culture conditions and isolation of genomic DNA}

Lactococcus lactis subsp. lactis IL1403 (kindly provided by M.P. Gaya, INIA, Madrid, Spain) and Streptococcus pneumoniae TIGR4 (purchased form the American Type Culture Collection) were used as the reference sequenced microorganisms. The test strain of Lactococcus garvieae used for the experiments was CECT 4531 (purchased from the Spanish Type Culture Collection). The L. lactis subsp. lactis IL1403 and L. garvieae CECT 4531 were grown statically at $28^{\circ} \mathrm{C}$ in $\mathrm{BHI}$ broth (bioMérieux, Marcy l'Etoile, France). The S. pneumoniae TIGR4 was grown statically at $37^{\circ} \mathrm{C}$ in Todd Hewitt broth (Oxoid, Basingstoke, Hampshire, England). Cells were grown until the late-exponential phase of growth (OD600 1.5-2) and harvested for isolation and purification of genomic DNA using the DNeasy Blood and Tissue kit (Qiagen, Hilden, Germany) according to the manufacturer's specifications. The DNA concentrations were determined spectrophotometrically.

\section{DNA labelling}

Aliquots $(1-2 \mu \mathrm{g})$ of genomic DNA from the three strains were labelled fluorescently with Cy3-dUTP or Cy5-dUTP (Perkin-Elmer, Foster City, CA, USA), depending on whether the strain was used as a test or reference microorganism in the $\mathrm{CGH}$ experiments, respectively. Each DNA aliquot was fragmented by sonication to obtain fragments from 400 to $1000 \mathrm{bp}$. Fragmented DNA was mixed with $5 \mu \mathrm{L} 10 \times$ NEBlot labelling buffer containing random sequence octamer oligonucleotides (New England Biolabs, Ipswich, MA, USA) and water to a final volume of $43.5 \mu \mathrm{L}$. This mixture was denatured by heating at $95^{\circ} \mathrm{C}$ for $5 \mathrm{~min}$ and then cooled for $5 \mathrm{~min}$ at $4^{\circ} \mathrm{C}$. After this denaturing step, the remaining components of the labelling reaction were added: $5 \mu \mathrm{L}$ of $10 \times$ dNTP labelling mix $(1.2 \mathrm{mM}$ each dATP, dGTP and dCTP in $10 \mathrm{mM}$ Tris pH 8.0, 1 mM EDTA) (New England Biolabs, Ipswich, MA, USA), $1.5 \mu \mathrm{L}$ of $1 \mathrm{mM} \mathrm{Cy3-dUTP}$ or Cy5-dUTP and $1.5 \mu \mathrm{L}$ of $10 \mathrm{U} / \mu \mathrm{L}$ Klenow fragment (Fermentas Life Sciences, Glen Burnie, $\mathrm{MD}, \mathrm{USA}$ ). The labelling reactions were incubated overnight at $37^{\circ} \mathrm{C}$ and then stopped by adding $2.5 \mu \mathrm{L}$ of 0.5 M EDTA. Labelled DNA was purified from unincorporated label using a Qiaquick PCR Cleanup kit (Qiagen, Hilden, Germany) and dried under vacuum. The final DNA concentration and quality, as well as the labelling quality, were determined using a NanoDrop (NanoDrop Techonologies, Wilmington, DE, USA).

\section{Array-based comparative genome hybridization (CGH)}

The L. lactis subsp. lactis IL1403 and S. pneumoniae TIGR4 microarrays used for the CGH analysis were purchased from Eurogentec (Serain, Belgium). The L. lactis microarray contains 4608 spots: 2126 duplicated ORFs, 32 negative controls and 324 empty spots. The S. pneumoniae microarray contains 4608 spots: 2087 duplicated ORFs, 224 negative controls and 210 empty spots.

The CGH experiments were performed by means of competitive hybridizations using DNA of $L$. lactis subsp. lactis IL1403 or S. pneumoniae TIGR4, depending on the array, as positive controls. The DNAs to be hybridized on the same array were labelled with Cy3-dUTP and Cy5dUTP, respectively. For each microarray hybridization reaction, aliquots $(1-2 \mu \mathrm{g})$ of labelled genomic DNAs of the reference (labelled with $\mathrm{Cy} 3$ ) and test (labelled with Cy5) strains, were mixed in $45 \mu \mathrm{L}$ EGT hybridization solution (Eurogentec, Serain, Belgium) and denatured at $65^{\circ} \mathrm{C}$ for $2 \mathrm{~min}$. The hybridization mixture was then loaded onto a microarray slide, covered with a coverslip and incubated at $38^{\circ} \mathrm{C}$ overnight. Following hybridization, the slides were washed in $2 \times \mathrm{SSC}, 0.5 \%$ SDS for $5 \mathrm{~min}$ followed by a second wash step in $1 \times$ SSC, $0.25 \%$ SDS for $5 \mathrm{~min}$. Finally, slides were rinsed in $0.2 \times$ SSC and dried by centrifugation.

The results presented herein represent a compilation of sixteen separate CGH experiments: L. lactis subsp. lactis IL1403 arrays (reference microorganism) were hybridized with S. pneumoniae TIGR4 (test microorganism) $(\mathrm{n}=2)$; 
S. pneumoniae TIGR4 arrays (reference microorganism) were hybridized with $L$. lactis subsp. lactis IL1403 (test microorganism) $(\mathrm{n}=2)$; L. lactis subsp. lactis IL1403 arrays (reference microorganism) were hybridized with $L$. garvieae CECT 4531 (test microorganism) $(\mathrm{n}=8) ; S$. pneumoniae TIGR4 arrays (reference microorganism) were hybridized with L. garvieae CECT 4531 (test microorganism) $(n=4)$. The data discussed in this publication have been deposited in NCBI's Gene Expression Omnibus [20] and are accessible through GEO Series accession number GSE19005. http://www.ncbi.nlm.nih.gov/geo/ query/acc.cgi?acc $=$ GSE19005.

\section{Data acquisition and analysis}

The microarray was scanned after hybridization using a Scanarray HT microarray scanner (Perkin-Elmer). The signal intensity of the two fluors was determined using ImaGene software (BioDiscovery, El Segundo, CA, USA). Microarray data were analysed using ImaGene software, Microsoft Excel and an in-house designed and built Microsoft Access database [21]. Gene calling was based on a signal-to-noise ratio (SNR) $>3$ for each spot. After the CGH experiments, a gene was considered to show a positive result when it was present in at least three of the four CGH assays. In the case of the L. garvieae CECT 4531 hybridizations with the L. lactis subsp. lactis IL1403 arrays, it was necessary to perform a larger number of assays $(n=8)$, owing to the poor quality of one of the batches of arrays used. Thus, the criterion chosen to determine a positive result in this case was when the gene was present in at least five of the eight CGH assays.

\section{In silico sequence analysis}

Sequence analyses were carried out to assess the performance of the inter-species CGH protocol. Using the BLAT [22] and BLAST [23] programs, the sequences of the $L$. lactis microarray probes were aligned with the $S$. pneumoniae genome sequence, and vice-versa. The BLAT search parameters were $90 \%, 80 \%$ and $70 \%$ sequence identity (BLAT90, BLAT80 and BLAT70) and a $100 \mathrm{bp}$ minimum alignment length (owing to the fact that the length of the array probe was between 100 and $400 \mathrm{bp}$ ). Available L. garvieae sequences of the nine previously identified genes that were positive in the CGH were aligned with the L. lactis subsp. lactis IL1403 or S. pneumoniae TIGR4 genomes and with the sequences of the immobilized probes of these genes in the corresponding microarray using BLAST [23] and BLAST 2 sequences [24] programs.

\section{Results}

\section{Inter-species comparison framework}

In silico analyses were performed to compare the sequences of the immobilized probes in the microarray of each reference organism with the sequences of their complete genomes available in GenBank (L. lactis subsp. lactis IL1403: NC_002662 and S. pneumoniae TIGR4: NC_003028). The BLAT alignment of the L. lactis IL1403 probes on the S. pneumoniae TIGR4 genome allowed the identification of 1 ORF with BLAT90, 65 ORFs with BLAT80 and 159 ORFs with BLAT70. Moreover, the BLAT alignment of the probes represented on the S. pneumoniae microarray on the L. lactis genome demonstrated 1 ORF, 63 ORFs and 165 ORFs for BLAT90, BLAT80 and BLAT70, respectively.

The CGH experiments based on swapping off the microarrays between S. pneumoniae and L. lactis identified 65 common ORFs. To evaluate the accuracy of the microarray $\mathrm{CGH}$ experiments, we compared these results with those of the in silico analysis. Out of the 65 genes, 47 (72\%) showed similarities greater than $80 \%, 16$ genes $(25 \%)$ exhibited a similarity between $70 \%$ and $80 \%$, and only 2 genes (3\%) showed a similarity slightly lower than $70 \%$ (66-68\%) (Table 1). In summary, 97\% of the genes detected by CGH showed similarities greater than $70 \%$ at the nucleotide level.

After combined analysis of the results obtained in silico and in vitro, we established, under the hybridization conditions used in this study, a detection threshold based on a sequence similarity of $\geq 70 \%$ for alignments longer than $100 \mathrm{bp}$. This was established as the reference framework for the inter-species CGH assays.

In vitro microarray CGH experiments with $L$. garvieae CECT 4531 vs reference microorganisms $L$. lactis subsp. lactis IL1403 and S. pneumoniae TIGR4, and in silico analysis of available sequences from $L$. garvieae

The microarray CGH experiments identified 267 genes in L. garvieae that had analogues in L. lactis and/or S. pneumoniae (Additional file 1). Of these, 111 genes (41.6\%) were identified only with the L. lactis microarray, 70 genes $(26.2 \%)$ only with the microarray of S. pneumoniae, and 86 genes $(32.2 \%)$ were identified with both microarrays. These genes belong to diverse functional groups (Table 2). Most of the genes (96.6\%) have been documented for the first time in L. garvieae. Only nine genes (four present in both reference microorganisms: atpD/SP1508, pfk/SP0896, tig/SP0400, tuf/ SP1489; three present in L. lactis: als, ddl, galK; two present in S. pneumoniae: SP0766, SP1219) out of the 267 genes detected have been either identified or sequenced before in diverse strains of $L$. garvieae (Tables 3 and 4). In silico analysis of these previously sequenced genes $(n=9)$ of L. garvieae were performed to assess the efficacy of the methodology. Alignments of these available sequences with the genomes of the corresponding reference microorganism and their respective array probes showed nucleotide identities ranging 
Table 1 In silico analysis of the common genes detected by CGH in the reference microorganisms

\begin{tabular}{|c|c|c|c|}
\hline CGH positive gene ID $^{a}$ & BLAT80 $^{b}$ & BLAT70 $^{\text {b, c }}$ & $\begin{array}{l}\text { \% Identity in } \\
\text { BLAST }^{\mathrm{d}}\end{array}$ \\
\hline atpD/SP1508 & ++ & & \\
\hline dnaG/SP1072 & - & - & $66 \%$ \\
\hline dnaJ/SP0519 & + & & \\
\hline dnaK/SP0517 & ++ & & \\
\hline enoA/SP1128 & ++ & & \\
\hline fbaA/SP0605 & ++ & & \\
\hline ftsZ/SP1666 & ++ & & \\
\hline fusA/SP0273 & ++ & & \\
\hline gapB/SP2012 & ++ & & \\
\hline gltX/SP2069 & - & ++ & \\
\hline $1 \mathrm{dh} / \mathrm{SP} 1220$ & ++ & & \\
\hline lepA/SP1200 & - & ++ & \\
\hline leuS/SP0254 & ++ & & \\
\hline lysS/SP0713 & - & ++ & \\
\hline msmk/SP1580 & ++ & & \\
\hline murA1/SP1081 & - & + & \\
\hline pepC/SP0281 & + & & \\
\hline pfk/SP0896 & - & + & \\
\hline pgiA/SP2070 & + & & \\
\hline pgk/SP0499 & ++ & & \\
\hline pmg/SP1655 & ++ & & \\
\hline ptnAB/SP0284 & + & & \\
\hline ptnD/SP0282 & - & + & \\
\hline$p t s / / S P 1176$ & - & ++ & \\
\hline purA/SP0019 & - & - & $76 \%$ \\
\hline pyk/SP0897 & ++ & & \\
\hline rpIASSP0631 & ++ & & \\
\hline$r p / B / S P 0212$ & ++ & & \\
\hline $\mathrm{rp} / \mathrm{C} / \mathrm{SP} 0209$ & ++ & & \\
\hline rplF/SP0225 & + & & \\
\hline rplJ/SP1355 & + & & \\
\hline rp/K/SP0630 & ++ & & \\
\hline rplL/SP1354 & ++ & & \\
\hline rp/M/SP0294 & ++ & & \\
\hline rplN/SP0219 & ++ & & \\
\hline rp/O/SP0229 & ++ & & \\
\hline rp/Q/SP0237 & + & & \\
\hline $\mathrm{rp} / \mathrm{S} / \mathrm{SP} 1293$ & ++ & & \\
\hline rp/V/SP0214 & + & & \\
\hline $\mathrm{rpm} / \mathrm{SP} 0960$ & + & & \\
\hline $\mathrm{rpm} / \mathrm{SPO233}$ & ++ & & \\
\hline rpoB/SP1961 & - & + & \\
\hline rpoC/SP1960 & + & ++ & \\
\hline rpoD/SP1073 & ++ & & \\
\hline rpsANSP0862 & - & + & \\
\hline$r p s B / S P 2215$ & - & ++ & \\
\hline$r p s C / S P 0215$ & ++ & & \\
\hline rpsD/SP0085 & ++ & & \\
\hline rpsE/SP0227 & ++ & & \\
\hline$r p s G / S P 0272$ & ++ & & \\
\hline
\end{tabular}

Table 1: In silico analysis of the common genes detected by CGH in the reference microorganisms (Continued)

\begin{tabular}{|c|c|c|c|}
\hline rpsJ/SP0208 & ++ & & \\
\hline rpsK/SP0235 & ++ & & \\
\hline rpsL/SP0271 & ++ & & \\
\hline rpsM/SP0234 & + & & \\
\hline rpsP/SP0775 & + & & \\
\hline rpsS/SP0213 & + & & \\
\hline topA/SP1263 & - & + & \\
\hline tuf/SP1489 & ++ & & \\
\hline typA/SP0681 & ++ & & \\
\hline upp/SP0745 & - & - & $77 \%$ \\
\hline ychH/SP2097 & + & & \\
\hline yciA/SP2096 & - & - & $68 \%$ \\
\hline yjiF/SP1565 & - & + & \\
\hline yjkl/SP2209 & - & + & \\
\hline yyaL/SP0004 & ++ & & \\
\hline \multicolumn{4}{|c|}{$\begin{array}{l}\text { erence organisms gene ID: Lactococcus lactis subsp. lactis IL1403/ } \\
\text { tococcus pneumoniae TIGR4 }\end{array}$} \\
\hline \multicolumn{4}{|c|}{$\begin{array}{l}\text { Genes detected in both alignments, L. lactis subsp. lactis IL1403 array } \\
\text { es vs S. pneumoniae TIGR4 genome, and S. pneumoniae TIGR4 array } \\
\text { es vs L. lactis subsp. lactis IL1403 genome; + positive in one of the two } \\
\text { s. }\end{array}$} \\
\hline
\end{tabular}

between $70 \%$ and $86 \%$ (Tables 3 and 4). Most of the available sequences (80\%) showed similarities greater than $75 \%$.

\section{Discussion}

In the present study, commercial microarrays of L. lactis subsp. lactis IL1403 and S. pneumoniae TIGR4 were used to determine the presence of homologous genes in L. garvieae. Both L. lactis and S. pneumoniae were chosen as reference organisms because they are closely related to $L$. garvieae $[18,19]$ and their genomes have been fully sequenced. Although these CGH experiments cannot detect and identify genes that are likely to exist only in the target microorganism, this approach reveals genes that are common to both the reference and the target organisms, allowing the identification of a large number of genes potentially present in an organism without the need for sequencing genomes $[17,25]$.

In experiments that involve inter-species comparison it is necessary to establish a framework that allows accurate comparison and interpretation of the results. Thus, the first efforts were focused on establishing that framework by the combination and integration of in silico analyses and in vitro microarray CGH experiments to compare the reference organisms $L$. lactis subsp. lactis IL1403 and S. pneumoniae TIGR4. Signal intensity has been used to assess the level of similarity between two genes in inter-species CGH experiments [15]. However, 
Table 2 Functional groups of genes identified in L. garvieae CECT 4531 according to the COG database

\begin{tabular}{|c|c|c|}
\hline Functional Group & $\begin{array}{l}\text { Homologous in L. lactis subsp. lactis } \\
\text { IL1403 }\end{array}$ & $\begin{array}{l}\text { Homologous in S. pneumoniae } \\
\text { TIGR4 }\end{array}$ \\
\hline Amino acid transport and metabolism & 14 & 10 \\
\hline Carbohidrate transport and metabolism & 24 & 15 \\
\hline Cell cycle control, cell division, cromosome partitioning & 4 & 2 \\
\hline Cell wall/membrane/envelope biogenesis & 5 & 4 \\
\hline Coenzime transport and metabolism & 1 & 1 \\
\hline DNA replication, recombination and repair & 8 & 12 \\
\hline Energy production and conversion & 11 & 6 \\
\hline Inorganic ion transport and metabolism & 4 & 5 \\
\hline Intracellular trafficking, secretion and vesicular transport & 4 & 2 \\
\hline Lipid transport and metabolism & 2 & 0 \\
\hline Nucleotide transport and metabolism & 15 & 11 \\
\hline Phage capside proteins & 1 & 0 \\
\hline $\begin{array}{l}\text { Post translational modification, protein turnover, } \\
\text { chaperones }\end{array}$ & 8 & 8 \\
\hline Signal transduction mechanisms & 2 & 3 \\
\hline Transcription & 7 & 6 \\
\hline Translation, ribosomal structure and biogenesis & 64 & 60 \\
\hline Unknown function & 23 & 11 \\
\hline Total & 197 & 156 \\
\hline
\end{tabular}

Table 3 In silico analysis of the available sequences of the genes detected in L. garvieae by CGH

\begin{tabular}{|c|c|c|c|c|}
\hline $\begin{array}{l}\text { Gene } \\
\text { ID }\end{array}$ & $\begin{array}{c}\text { GenBank accession number of } L . \text { garvieae } \\
\text { sequence }\end{array}$ & L. garvieae strain & $\begin{array}{c}\text { Similarity with L. lactis subsp. lactis } \\
\text { IL1403 gene (\%) }\end{array}$ & $\begin{array}{c}\text { Similarity with array } \\
\text { probe (\%) }\end{array}$ \\
\hline als & EF450031 & UNIUD074 & 77 & 76 \\
\hline $\operatorname{atpD}$ & AX111128 & $\begin{array}{l}\text { from patent } \\
\text { W00123604 }\end{array}$ & 86 & 86 \\
\hline$d d l$ & AF170808 & E. serolicida & 72 & 75 \\
\hline galk & EU153555 & DSM 20684 & 78 & 79 \\
\hline$p f k$ & AB024532 & SA8201 & 85 & 84 \\
\hline tig & AB024531 & SA8201 & 82 & - \\
\hline tuf & AX109994 & $\begin{array}{l}\text { from patent } \\
\text { W00123604 }\end{array}$ & 80 & 77 \\
\hline
\end{tabular}

Results for the L. lactis subsp. lactis IL1403 array based-CGH

this approach may be influenced, and therefore biased, by different factors, such as regional sample labelling effects, probe accessibility or local hybridization issues [13]. For these reasons, in the present study signal intensity was not considered for determining whether a gene was positive or not in the inter-species $\mathrm{CGH}$ experiments.

These analyses revealed that nearly all the genes common to L. lactis and S. pneumoniae that were detected by swap microarray CGH experiments (97\%) exhibited a sequence similarity of at least $70 \%$ (Table 1 ). Only two genes ( $d n a G$ and $y c i A)$ detected in the microarray CGH experiments showed a sequence similarity slightly lower than $70 \%$ (66 and 68\%, respectively; Table 1). Variability in the factors that influence the CGH signals, such as systematic errors (e.g. dye effects), copy number variation, and sequence divergence between the analysed samples [13], may explain these results. The comparison of the results of both analyses, in silico and in vitro, for the reference microorganisms (Table 1) allowed us to establish that, under our experimental conditions, it was possible to detect and identify inter-species hybridization with a detection threshold based on a sequence similarity of $\geq 70 \%$.

Therefore, our threshold value of sequence similarity $\geq 70 \%$ was set up directly from the comparison of the results of the in silico and in vitro analyses of the present study. This threshold value was used subsequently to interpret the results of the microarray-based CGH experiments comparing L. garvieae and the reference microorganisms. Less stringent hybridization conditions would probably have allowed the identification of a larger number of genes, but this would have also resulted in lower specificity. Given that the final aim of the 
Table 4 In silico analysis of the available sequences of the genes detected in L. garvieae by CGH

\begin{tabular}{|c|c|c|c|c|}
\hline $\begin{array}{l}\text { Gene } \\
\text { ID }\end{array}$ & $\begin{array}{c}\text { GenBank accession number of } L \text {. garvieae } \\
\text { sequence }\end{array}$ & L. garvieae strain & $\begin{array}{c}\text { Similarity with S. pneumoniae TIGR4 } \\
\text { gene (\%) }\end{array}$ & $\begin{array}{c}\text { Similarity with array } \\
\text { probe (\%) }\end{array}$ \\
\hline SP1508 & AX111128 & $\begin{array}{l}\text { from patent } \\
\text { WO0123604 }\end{array}$ & 82 & 82 \\
\hline SP0896 & AB024532 & SA8201 & 80 & 79 \\
\hline \multirow[t]{3}{*}{ SP0766 } & AM490328 & JIP 31-90 (2) & 71 & 79 \\
\hline & AJ387925 & CIP 102507 T & 70 & 70 \\
\hline & AJ387923 & $\begin{array}{l}\text { E. serolicida } \\
\text { ATCC49156 }\end{array}$ & 70 & 70 \\
\hline SP04000 & AB024531 & SA8201 & 74 & - \\
\hline SP1489 & AX109994 & $\begin{array}{l}\text { from patent } \\
\text { W00123604 }\end{array}$ & 80 & 79 \\
\hline \multirow[t]{11}{*}{ SP1219 } & AB364641 & 20-92 & 84 & 86 \\
\hline & AB364640 & Lc. 1236 & 84 & 85 \\
\hline & AB364639 & LC. 925 & 85 & 85 \\
\hline & AB364638 & LC. 881 & 84 & 84 \\
\hline & AB364637 & LC. 337 & 85 & 85 \\
\hline & AB364633 & LMG9472 & 85 & 85 \\
\hline & AB364632 & ATCC43921 & 84 & 84 \\
\hline & AB364627 & G50202 & 84 & 84 \\
\hline & AB364626 & KGLA5224 & 84 & 84 \\
\hline & AB364625 & EH5803 & 83 & 83 \\
\hline & AB364624 & KG9408 & 84 & 84 \\
\hline
\end{tabular}

Results for the S. pneumoniae TIGR4 array based-CGH

experiment was the identification of genes potentially present in $L$ garvieae, it was preferred to maintain stringent hybridization conditions, therefore increasing the specificity and the reliability of the results. Hence, the genes detected in the $\mathrm{CGH}$ experiments should have an analogue in L. garvieae with a nucleotide sequence identity greater than $70 \%$ with the respective gene in the reference organism.

The CGH hybridizations using L. lactis subsp. lactis IL1403 and $S$. pneumoniae TIGR4 microarrays identified 267 analogous genes in L. garvieae (Additional file 1). Only $3.4 \%$ of these genes (nine out of 267) have been characterized or sequenced previously by other groups in different strains of L. garvieae [[18,26-29], and GenBank sequences: AX109994, AB364624, AB364625, AB364626, AB364627, AB364632, AB364633, AB364637, AB364638, AB364639, AB364640, AB364641, EU153555]. The alignments of the available sequences of these nine previously of these nine previously identified genes in L. garvieae with both the sequences of these genes from the reference microorganisms and those from the array probe showed nucleotide similarities greater than 70\% (70-86\%) between them (Tables 3 and 4). These data are consistent with the detection threshold value discussed previously. Therefore it is reasonable to assume that the other genes detected in L. garvieae CECT 4531 by CGH experiments will also have at least $70 \%$ sequence similarity with the respective genes in the reference microorganisms. The positive result obtained in both CGH experiments for the tig/SP0400 gene (Tables 3 and 4), was unexpected given the absence of similarity between the available sequence and the probes on both microarrays. This result could be explained by the fact that the available sequence for L. garvieae is partial, and it represents a part of the gene that does not correspond with the probe.

We classified the ORFs into clusters of orthologous genes (COGs) [30]. The 267 genes identified in L. garvieae CECT 4531 (Additional file 1) belong to diverse biological functional groups (Table 2). Most of the genes detected in L. garvieae (about 66\%) were related to meaningful biological functions such as those related to ribosomal functions, sugar metabolism or energy conversion systems, which are usually represented in Lactobacillales [31]. The remaining genes identified included "housekeeping genes", such as $\operatorname{gyr} B$, $\operatorname{sod} A, \operatorname{rec} A$, ileS, $r p o D$, dnaK and $d d l$ [19], genes of diverse functional groups and genes with unknown functions. Some of them are of interest because they could be involved in the pathogenesis of L. garvieae infections. For example, the gene als, which has been described as an important factor for host colonization by El Tor biotypes of Vibrio cholerae [32], has also been suggested to be one of the genes required for survival of L. garvieae in fish [27]. In addition, the gene $m y c A$, which was detected for the first time in L. garvieae in the present study, encodes an antigen that cross-reacts with myosin, and members of 
this family of proteins have been suggested to play an important role in the pathogenesis of streptococcal infections [33].

Sequencing of the genes identified in this work is beyond the scope of this initial study, but the data provided can be the starting point for future genetic analysis of $L$. garvieae strains from different ecological niches or adapted to different host species.

This study provides the first insight into the genome content of L. garvieae and suggests that CHG could be a useful approach for studying the genetic content of other Gram-positive catalase-negative cocci of human and veterinary relevance.

\section{Conclusions}

In the present work, a comparative analysis based on microarray interspecies hybridization and on the use of bioinformatic tools was used for the first time to study the genetic content of L. garvieae CECT 4531. It is important to remark that the integration of results from bioinformatics and microarray-based CGH requires the definition of a framework that allows an accurate comparison and interpretation of the results obtained. Once this framework was established, it was possible to identify 267 genes potentially present in L. garvieae CECT 4531. Some of the identified genes, such as the als and $m y c A$ genes, could be involved in the pathogenesis of $L$. garvieae infections.

In summary, these results provide the first insight into the genome content of L. garvieae and could be useful for future understanding of the genetics of this pathogenic microorganism.

Additional file 1: Genes potentially identified in L. garvieae CECT 4531 and their homologues in L. lactis subsp. lactis IL1403 and S. pneumoniae TIGR4.

\section{Acknowledgements}

This work was supported partially by projects AGL2005-04775 and AGL200912447 of the Ministerio Español de Ciencia e Innovación. M. Aguado-Urda was a recipient of a grant from Centro de Vigilancia Sanitaria Veterinaria (VISAVET), and a PhD grant from the Universidad Complutense de Madrid. The work of Dr. López-Campos and Dr. Martín-Sanchez was partially funded by the COMBIOMED Network and ONTOMINEBASE reseach project (Ministerio Español de Ciencia e Innovación). The authors thank M.P. Gaya for providing the Lactococcus lactis subsp lactis IL1403 strain.
}

\section{Author details \\ 'Departamento de Sanidad Animal, Facultad de Veterinaria, Universidad Complutense, 28040 Madrid, Spain. Área de Bioinformática y Salud Pública, Instituto de Salud Carlos III, 28220 Madrid, Spain. ${ }^{3}$ Centro de Vigilancia Sanitaria Veterinaria (VISAVET), Facultad de Veterinaria, Universidad Complutense, 28040 Madrid, Spain.}

\section{Authors' contributions}

MAU carried out the microarray experiments and the bioinformatics analyses, and participated in the analysis of the data and drafting of the manuscript. GHLC designed the microarray experiments, and participated in analysis of the data and drafting of the manuscript. JFFG participated in and supervised drafting of the manuscript. FMS participated in and supervised the design of the microarray experiments and the analysis of the microarray data. AG participated in the design of the study and drafting of the manuscript. LD participated in the design of the study. MB conceived the study, and participated in its design and coordination. All the authors read and approved the final manuscript.

Received: 3 December 2009 Accepted: 16 March 2010 Published: 16 March 2010

\section{References}

1. Chen SC, Liaw LL, Su HY, Ko SC, Wu CY, Chaung HC, Tsai YH, Yang KL, Chen YC, Chen TH, Lin GR, Cheng SY, Lin YD, Lee JL, Lai CC, Weng YJ, Chu SY: Lactococcus garvieae, a cause of disease in grey mullet, Mugil cephalus, in Taiwan. J Fish Dis 2002, 25:727-732.

2. Vela Al, Vázquez J, Gibello A, Blanco MM, Moreno MA, Liébana $P$, Albendea C, Alcalá B, Méndez A, Domínguez L, Fernández-Garayzábal JF: Phenotypic and genetic characterization of Lactococcus garvieae isolated in Spain from lactococcosis outbreaks and comparison with isolates of other countries and sources. J Clin Microbiol 2000, 38:3791-3795.

3. Vendrell D, Balcázar JL, Ruiz-Zarzuela I, de Blas I, Gironés O, Múzquiz JL: Lactococcus garvieae in fish: a review. Comp Immunol Microbiol Infect Dis 2006, 29:177-198.

4. Evans JJ, Pasnik DJ, Klesius PH, Al-Ablani S: First report of Streptococcus agalactiae and Lactococcus garvieae from a wild bottlenose dolphin (Tursiops truncatus). J Wild Dis 2006, 42:561-569.

5. Collins MD, Farrow JAE, Phillips BA, Kandler O: Streptococcus garvieae sp. nov. and Streptococcus plantarum sp. J Gen Microbiol 1983, 129:3427-3431.

6. Teixeira LM, Merquior VLC, Vianni MCV, Carvalho MGS, Fracalanzza SEL, Steigerwalt AG, Brenner DJ, Facklam RR: Phenotypic and genotypic characterization of atypical Lactococcus garvieae strains isolated from water buffalos with subclinical mastitis and confirmation of $L$ garvieae as a senior subjetive synonym of Enterococcus seriolicida. Int J Syst Bacteriol 1996, 46:664-668.

7. Pot B, Devriese LA, Ursi D, Vandamme P, Haesebrouck F, Kersters K: Phenotypic identification and differentiation of Lactococcus strains isolated from animals. Syst Appl Microbiol 1996, 19:213-222.

8. Elliot JA, Collins MD, Pigott NE, Facklam RR: Differentiation of Lactococcus lactis and Lactococcus garvieae from humans by comparison of wholecell protein patterns. J Clin Microbiol 1991, 20:2731-2734.

9. Facklam RR, Elliot JA: Identification, classification, and clinical relevance of catalase-negative, Gram-positive cocci, excluding the streptococci and enterococci. Clin Microbiol Rev 1995, 8:479-495.

10. Fefer JJ, Ratzan KR, Sharp SE, Saiz E: Lactococcus garvieae endocarditis: report of a case and review of the literature. Diagn Microbiol Infect Dis 1998, 32:127-130.

11. Li WK, Chen YS, Wann SW, Liu YC, Tsai HT: Lactococcus garvieae endocarditis with initial presentation of acute cerebral infarction in a healthy immunocompetent man. Inter Med 2008, 47:1143-1146.

12. Wang CY, Shie HS, Chen SC, Huang JP, Hsieh IC, Wen MS, Lin FC, Wu D: Lactococcus garvieae infections in humans: possible association with aquaculture outbreaks. Int J Clin Pract 2007, 61:68-73.

13. van Hijum SAFT, Baerends RJS, Zomer AL, Karsens HA, Martín-Requena V, Trelles O, Kok J, Kuipers OP: Supervised Lowess normalization of comparative genome hybridization data-application to lactococcal strain comparisons. BMC Bioinf 2008, 9:93.

14. Hakenbeck R, Balmelle N, Weber B, Gardes C, Keck W, de Saizieu A: Mosaic genes and mosaic chromosomes: intra- and interspecies genomic variation of Streptococcus pneumoniae. Infect Immun 2001, 69:2477-2486.

15. Dong Y, Glasner JD, Blattner FR, Triplett EW: Genomic interspecies microarray hybridization: rapid discovery of three thousand genes in the maize endophyte, Klebsiella pneumoniae 342 , by microarray hybridization with Escherichia coli K-12 Open Reading Frames. Appl Environ Microbiol 2001, 67:1911-1921.

16. Fukiya $\mathrm{S}$, Mizoguchi H, Tobe T, Mori H: Extensive genomic diversity in pathogenic Escherichia coli and Shigella strains revealed by comparative genomic hybridization microarray. J Bacteriol 2004, 186:3911-3921.

17. Zhang L, Reddi U, Srinivasan U, Li S, Borchardt SM, Pillai P, Mehta P, Styka AN, DeBusscher J, Marrs CF, Foxman B: Combining microarray technology and molecular epidemiology to identify genes associated 
with invasive group B Streptococcus. Interdiscip Perspect Infect Dis 2008, 2008:314762.

18. Michel C, Pelletier C, Boussaha M, Douet DG, Lautraite A, Tailliez P: Diversity of lactic acid bacteria associated with fish and the fish farm environment, established by amplified rRNA gene restriction analysis. Appl Environ Microbiol 2007, 73:2947-2955.

19. Coenye T, Vandamme P: Extracting phylogenetic information from wholegenome sequencing projects: the lactic acid bacteria as a test case. Microbiol 2003, 149:3507-3517.

20. Edgar R, Domrachev M, Lash AE: Gene Expression Omnibus: NCBI gene expression and hybridization array data repository. Nucleic Acids Res 2002, 30:207-210.

21. López-Campos GH, García-Albert L, Martín-Sánchez F, García-Sáez A: Analysis and management of HIV peptide microarray experiments. Methods Inf Med 2006, 45:158-162.

22. Kent WJ: BLAT-the BLAST-like alignment tool. Genome Res 2002, 12:656-664.

23. Altschul SF, Gish W, Miller W, Myers EW, Lipman DJ: Basic local alignment search tool. J Mol Biol 1990, 215:403-410.

24. Tatusova TA, Maden TL: BLAST 2 Sequences, a new tool for comparing protein and nucleotide sequences. FEMS Microbiol Lett 1999, 2:247-250.

25. Zhang $R$, Zhang $C T$ : The impact of comparative genomics on infectious disease research. Microbes and Infect 2006, 8:1613-1622.

26. Hirono I, Yamashita H, Park Cl, Yoshida T, Aoki T: Identification of genes in a KG - phenotype of Lactococcus garvieae, a fish pathogenic bacterium whose proteins react with antiKG-rabbit serum. Microb Pathog 1999, 27:407-417.

27. Menéndez A, Fernández L, Reimundo $P$, Guijarro JA: Genes required for Lactococcus garvieae survival in a fish host. Microbiology 2007, 153:3286-3294

28. Ozawa Y, Courvalin P, Gaiimand M: Identification of enterococci at the species level by sequencing of the genes for D-alanine: D-alanine ligases. Syst Appl Microbiol 2000, 23:230-237.

29. Poyart C, Quesnes G, Trieu-Cuot P: Sequencing the gene encoding manganese dependent superoxide dismutase for rapid species identification of enterococci. J Clin Microbiol 2000, 38:415-418.

30. Tatusov RL, Galperin MY, Natale DA, Koonin EV: The COG database: a tool for genome-scale analysis of protein functions and evolution. Nucleic Acids Res 2000, 1:33-36.

31. Makarova K, Slesarev A, Wolf Y, Sorokin A, Mirkin B, Koonin E, Pavlov A, Pavlova N, Karamychev V, Polouchine N, Shakhova V, Grigoriev I, Lou Y, Rohksar D, Lucas S, Huang K, Goodstein DM, Hawkins T, Plengvidhya V, Welkeri D, Huges J, Goh Y, Benson A, Baldwin K, Lee JH, Díaz-Muñiz I, Dosti B, Smeianov V, Wechter W, Barabote R, Lorca G, Altermann E, Barrangou R, Ganesan B, Xie Y, Rawsthorne H, Tamir D, Parker C, Breidt F, Broadbent J, Hutkins R, O'Sullivan D, Steele J, Unlu G, Saier M, Klaenhammer T, Richardson P, Kozyavkin S, Weimer B, Mills D: Comparative genomics of the lactic acid bacteria. PNAS 2006, 103:15611-15616.

32. Yoon SS, Mekalanos JJ: 2,3-butanediol synthesis and the emergence of the Vibrio cholerae El Tor biotype. Infect Immun 2006, 74:6547-6556.

33. Kil KS, Cunningham MW, Barnett LA: Cloning and sequence analysis of a gene encoding a 67-kilodalton myosin-cross-reactive antigen of Streptococcus pyogenes reveals its similarity with class II major histocompatibility antigens. Infect Immun 1994, 62:2440-2449.

doi:10.1186/1471-2180-10-79

Cite this article as: Aguado-Urda et al:: Analysis of the genome content of Lactococcus garvieae by genomic interspecies microarray hybridization. BMC Microbiology 2010 10:79.

\section{Submit your next manuscript to BioMed Central and take full advantage of:}

- Convenient online submission

- Thorough peer review

- No space constraints or color figure charges

- Immediate publication on acceptance

- Inclusion in PubMed, CAS, Scopus and Google Scholar

- Research which is freely available for redistribution

Submit your manuscript at www.biomedcentral.com/submit
Biomed Central 